\title{
Inexpensive Method of Liposuction Cannula Port-Site Protection
}

\author{
Jonathan Bank • David H. Song
}

Received: 30 January 2013/Accepted: 12 April 2013/Published online: 10 May 2013

(C) Springer Science+Business Media New York and International Society of Aesthetic Plastic Surgery 2013

Level of Evidence $V$ This journal requires that authors assign a level of evidence to each article. For a full description of these Evidence-Based Medicine ratings, please refer to the Table of Contents or the online Instructions to Authors www.springer.com/00266.

Suction-assisted lipoplasty is a frequently used technique in plastic surgery. One of the common side effects is skin damage at the port sites resulting from friction endured during use. Further damage may result from contact with a hot ultrasound-assisted liposuction probe. Several skin protectors are available on the market at varying costs ranging from $\$ 10$ to $\$ 20$.

We hereby propose the use of a truncated 1-ml plastic syringe, readily available in any operating room or office setting. The syringe plunger is removed, and the needle hub is cut off at an angle to facilitate introduction into the skin incision (Fig. 1). Suction cannulas up to $4 \mathrm{~mm}$ in diameter can be passed easily through the barrel. The syringe flanges may be sutured to the skin to prevent inadvertent dislodgement during the liposuction (Fig. 2).

Given the low cost (roughly $\$ 0.20$ per syringe), widespread availability, and ease of use, we offer this impromptu contraption as a simple adjunct to routine surgical use.

J. Bank ( $\varangle)$ · D. H. Song

Section of Plastic and Reconstructive Surgery,

Department of Surgery, University of Chicago Medical Center, 5841 S. Maryland Avenue, MC 6035, Chicago, IL 60637, USA e-mail: bank.jonathan@gmail.com

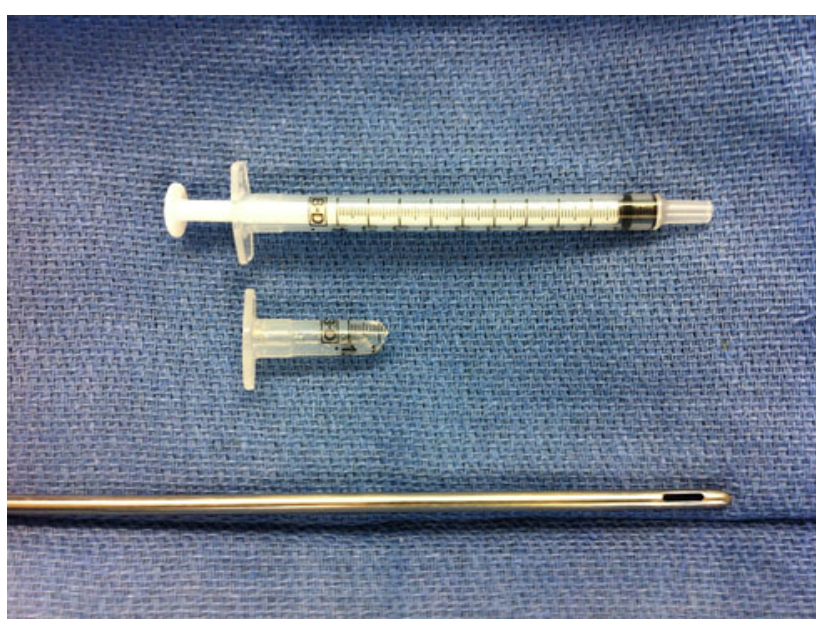

Fig. 1 A 1-ml syringe truncated syringe enables introduction of a 4-mm suction cannula

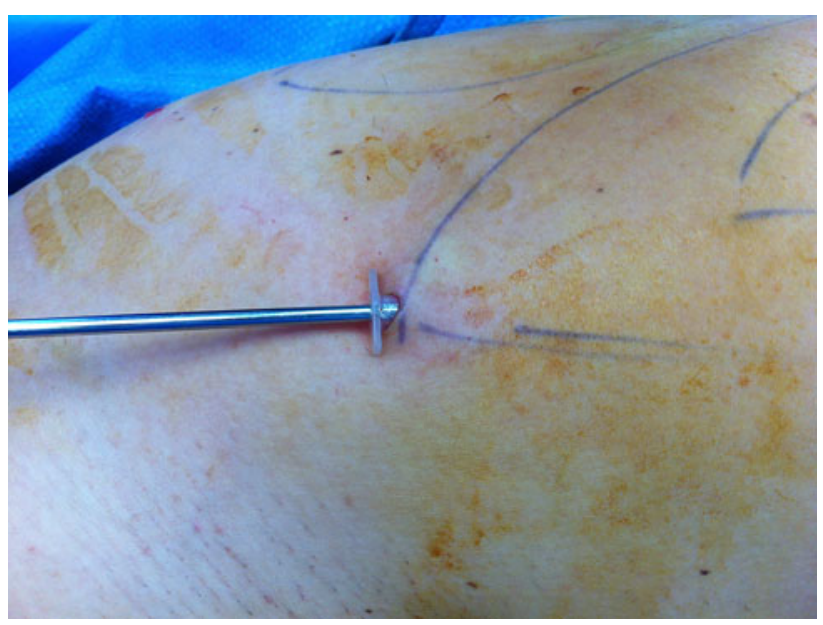

Fig. 2 The truncated syringe is introduced into a stab incision at the selected site, and the suction cannula is passed through the opening 